\title{
Spectral and 3-Dimensional Tracking of Single Gold Nanoparticles in Living Cells Studied by Rayleigh Light Scattering Microscopy ${ }^{\dagger}$
}

\author{
Guillaume Louit, \\ Department of Applied Physics, Osaka University, Suita, Osaka 565-0871, Japan, Graduate School of \\ Materials Science, Nara Institute of Science and Technology, Ikoma, Nara 630-0192, Japan, and Department of \\ Applied Chemistry and Institute of Molecular Science, National Chiao Tung University, \\ Hsinchu 30010, Taiwan
}

Received: February 27, 2009; Revised Manuscript Received: April 29, 2009

\begin{abstract}
Using a confocal Rayleigh light scattering microspectroscopy and imaging system, we followed and recorded the scattering spectra of single gold nanoparticles interacting with living mouse fibroblast cells, during the scale of $1 \mathrm{~h}$. A white light continuum produced by a femtosecond laser was focused on gold nanoparticles with a diameter of $80 \mathrm{~nm}$, while an incubation system ensured viability of the living cells. After reporting spectral characteristics of gold nanoparticles and their target fibroblasts, separately and in combination, tracking sequences of single nanoparticles in contact with a single cell are described. Through the study of three separate and representative cases, transitory as well as plasmon resonance band shifts, which can be linked to biological events, have been observed and are discussed here.
\end{abstract}

\section{Introduction}

New labels in bioanalysis which are stable and easily functionalized are becoming increasingly important. Among them, plasmon resonant particles followed by light scattering are an interesting alternative to fluorescent molecules and quantum dots, as the latter are unfortunately subject to important drawbacks such as bleaching and blinking. Light scattering by noble metal nanoparticles is dominated by the resonance between the collective oscillations of electrons in the conduction band and the incident light electric field. ${ }^{1-4}$ The scattered light color under white illumination is the result of the particle plasmon resonance, its band peak depending on size, shape, and environment of the particles and their mutual interaction. ${ }^{5-15}$ Silver and gold nanoparticles have been used as common probes for decades in the biological field but more recently new applications have been found, such as in vitro biosensors. ${ }^{16-22}$ Yet, gold nanoparticles as in vivo sensors or therapeutic agents constitute an even greater challenge and have therefore started to attract much attention during the past few years, usually as bioconjugates. ${ }^{23}$ Among them, two achievements worth mentioning are antibody-modified gold particles for cancer detection and therapy ${ }^{24-30}$ and controlled DNA delivery. ${ }^{31-37}$

There have been extensive studies on the effect of environmental parameters on the light scattering properties of noble metal nanoparticles. Two main kinds of plasmon resonance peak shifts are under interest for bioapplications: the shift that occurs when two (or more) particles mutually interact, and the shift that occurs when the surrounding environment of a single particle undergoes a significant change. Bioassays based on pair or aggregate formation are sometimes based on DNA triggered aggregation, ${ }^{38}$ where the wavelength shift is very large and easily followed by using colorimetric methods. ${ }^{16,17,39}$ Other nanopar-

\footnotetext{
" Part of the "Hiroshi Masuhara Festschrift".

* To whom correspondence should be addressed. Fax: +81 668797840 Phone: +81 66879 7839. E-mail: asahi@ap.eng.osaka-u.ac.jp.

¥ Osaka University.

"National Chiao Tung University.

${ }^{\S}$ Nara Institute of Science and Technology.
}

ticles aggregation bioassays include methods to detect antiprotein $\mathrm{A} .{ }^{40}$ Although the most noticeable changes are reported during pair formations, ${ }^{15,41}$ Haes et al. reported a peak wavelength shift of more than $30 \mathrm{~nm}$ for functionalized silver nanoparticles in an antigen-antibody sandwich assay. ${ }^{18,20}$ In other cases, peak wavelength shifts are as small as a few nanometer order and thus highly sensitive detection techniques are required..$^{42}$ On the other hand, spherical gold nanoparticles (50 to $130 \mathrm{~nm}$ in diameter) in PVA films of varying thickness studied at a single particle level in our group have shown the spectral peak shift of a few tens of nanometers when increasing the thickness from 30 to $330 \mathrm{~nm}$, proving that these nanoparticles are influenced by their surrounding environment to the extent of $100 \mathrm{~nm}^{10}$

Little is known regarding the response of the scattering properties of gold nanoparticles in vivo, the main reason being the experimental difficulties involved. One group reported a 16 $\mathrm{nm}$ shift of the plsamon resonance band when $35 \mathrm{~nm}$ gold particles are put into contact with cells, ${ }^{26}$ but it must be pointed out that light scattering measurements were ensemble average. Nanoparticle aggregation might be attributed to the large peak shift. To our knowledge, there are no data on the evolution of the spectra of a single nanoparticle in contact with a living cell. To obtain the necessary information, the same particle must be followed over time, so it is necessary to perform both particle tracking and spectrum acquisition.

Tracking of single particles such as fluorophores or quantum dots in biological systems has received much attention in the last two decades, and was mainly designed for the purpose of measuring diffusion coefficients, ${ }^{43}$ identification of targets through mobility, ${ }^{44}$ and particle motion mechanisms. ${ }^{45-47}$ Noble metal nanoparticles have been less studied by tracking experiments. Xu et al. have tracked silver enhanced gold nanoparticles and measured their uptake and release dynamics by bacteria using dark field conventional microscopy, ${ }^{48,49}$ Murase et al. have studied diffusion of lipid-labeled $40 \mathrm{~nm}$ gold nanoparticles on membrane with bright field microscopy, ${ }^{50}$ and Yelin et al. have used multiphoton plasmon resonance to locate gold nanoparticles 


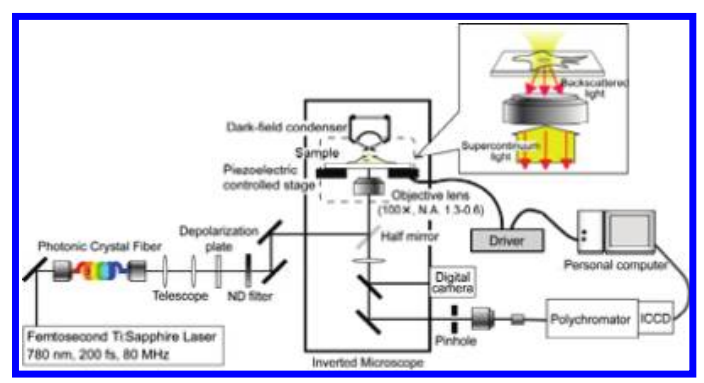

Figure 1. A schematic diagram of our confocal light scattering microspectroscopy and imaging system.

in living cells. ${ }^{51}$ By using a photothermal imaging method, the size boundaries of tracking have been recently pushed down to $5 \mathrm{~nm} \cdot{ }^{52-55} \mathrm{New}$ and very promising techniques that can achieve spectroscopic measurements of small nanopaticles have not yet been applied to single biological cells. The detection and tracking capabilities of the interferometry-based method of Sandoghdar et al. is of particular interest. ${ }^{56,57}$

We were interested in the evolution of spectral properties of single gold nanoparticles inside cells. Our tracking methods were therefore not destined to equal the highest positioning accuracies already reached by single particle tracking, nor to aim for the best time resolution. Recently, we developed a confocal Rayleigh light scattering microspectroscopy and imaging system, using femtosecond supercontinuum as a probe light, which archives a broadband $(500-750 \mathrm{~nm})$ light scattering spectral measurement with a submicrometer 3-D spatial resolution (vertical: $300 \mathrm{~nm}$; horizontal: $800 \mathrm{~nm}$ ).$^{58}$ This system will have both spectral properties and positioning, which allows a sufficient time resolution to monitor endocytocis/exocytosis phenomena. We report here for the first time variations of the spectral properties of individual $80 \mathrm{~nm}$ gold nanoparticles in interaction with living cells, on the time scale of the endocytic process.

\section{Experimental Methods}

Light Scattering Spectroscopic System. Figure 1 shows a schematic illustration of our confocal light scattering microspectroscopy and imaging system. The system is based on a confocal microscope setup (IX-70, Olympus) in which a strongly directional white light continuum, generated by focusing the output of a mode-locked fs-Ti:Sapphire laser (Tsunami 3941-T1AW Spectraphysics, $780 \mathrm{~nm}$ center wavelength, $120 \mathrm{fs}$ pulse width, $80 \mathrm{MHz}$ repetition rate, $800 \mathrm{~mW}$ power) into a photonics crystal fiber (NL-PL-750, Crystal Fiber, $1.8 \mu \mathrm{m}$ core diameter, $750 \mathrm{~nm}$ zero dispersion wavelength, $100 \mathrm{~cm}$ length), is used as a probe light. After passing through a filter (constituted of a $40 \mathrm{~nm}$ gold nanoparticles solution we prepared) to obtain a more homogeneous spectrum in the $450-750 \mathrm{~nm}$ range, this light beam is focused on the sample constituted of cells and/or nanoparticles, using an oil immersion objective (UPlanFl, Olympus: $\times 100 ;$ N.A. 1.3$)$. Then, the light scattering spectrum of each particle can be recorded, from 450 to 750 $\mathrm{nm}$, by collecting the back-scattered light from samples with the same objective lens and introduced, after an imaging pinhole, into a polychromator (77480, ORIEL) coupled with an image-intensified charge-coupled device (ICCD; iStar DH720-18, Andor Technology). To convert the scattered light into scattering light efficiency, we divided the measured intensity of scattered light from samples by that of reflection light from the surface of a coverslip that has uniform reflectance in the spectral range of our experimental setup.

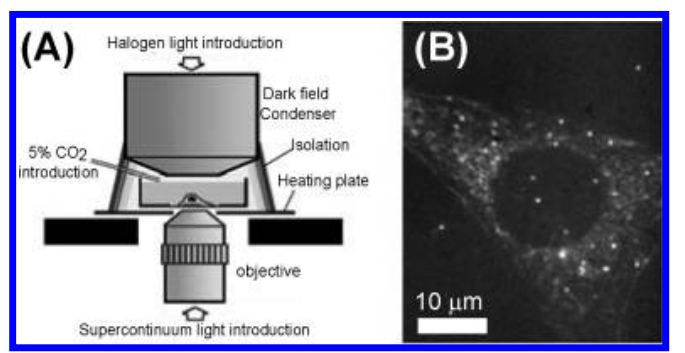

Figure 2. (A) Schematic diagram of the microscope stage of the experimental setup. (B) A typical dark field image of a mouse NIH3T3 fibroblast after gold nanoparticle injection and mild agitation of the medium.

A sample was set on a piezo electric controlled 3-D stage (Nano LP-100, Mad City Laboratories.) that was mounted on a XY motorized microscope stage (BIOS-102T, Sigma Koki). By scanning the piezo stage position, which was synchronized to the scattering light measurement with the ICCD polychromator, we obtained the scattering spectra at each point of the target and then constructed spectroscopic images. The spatial resolution was examined by measuring 3-D sccaning images of a single gold nanoparticle (100 $\mathrm{nm}$ diameter) on a coverslip covered with immersion oil. The lateral and axial spatial resolutions were evaluated to be 300 and $800 \mathrm{~nm}$ respectively from fwhm (full width at half-maximum) of the sectional intensity profiles of the image. Conventional dark field images of the cells were also recorded by using the same objective (with N.A. reduced to 0.6 ) and halogen illumination through a dark field condenser (Olympus U-EDC W).

Gold Nanoparticles. Concerning the particle size, we have chosen to study $80 \mathrm{~nm}$ spherical gold particles as their light scattering can be more easily distinguished from the background. The cell scattering signal systematically displays interferences which are the major factor limiting sensitivity of the setup. Because the light scattering intensity of nanoparticles decreases drastically with a decrease of the particle size, the minimal size for the spectral tracking by the present setup is about $50 \mathrm{~nm}$ in diameter. In addition, $80 \mathrm{~nm}$ particles have been proven to be endocytozed by mammalian cells ${ }^{59}$ and citrate capped gold nanoparticles are not cytotoxic to cultivated cells. ${ }^{60}$ As for particle concentration, a stock colloidal suspension (EMGC80, British BioCell) was diluted to $1 \mathrm{pM}$ when in contact with cells. Thus, there are $6 \times 10^{8}$ particles in the sample volume, which corresponds to about 1 particle on a $10 \mu \mathrm{m}$ box. We could observe single nanoparticles by eye in a typical experiment, and confirmed only a few particles entered the vicinity of a cell during experiments.

Biological Cells. Mouse NIH-3T3 fibroblasts were plated onto a glass bottom dish (Matsunami, thickness of $0.08-0.12 \mathrm{~mm}$ ) previously coated with fibronectin from Human Plasma (Calbiochem) and cultured in Dulbecco's Modified Eagle's Medium (Sigma Aldrich). Immediately after being set on the microscope stage, the medium was replaced by a gold colloidal solution diluted in PBS buffer (Aldrich). Through dark field illumination, we record images of the cell, to check if it changes position and/or shape during the course of the experiment or not.

Cell Observation and Particle Tracking Methodology. To offer the best environmental conditions for mouse fibroblast cells, we introduced a heating system to keep the cells at 37.5 ${ }^{\circ} \mathrm{C}$ and a $\mathrm{CO}_{2}$ incubation system (Figure $2 \mathrm{~A}$ ). These modifications allowed us to retain both laser and dark field illumination, but more importantly, the cell stability with time was significantly improved and experiments could be conducted beyond $1 \mathrm{~h}$. A triangular shape cell was chosen using halogen light dark 


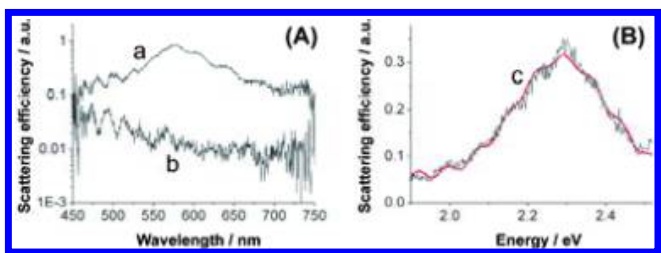

Figure 3. (A) Typical scattering spectra of gold nanoparticles in contact with a living cell (a), and nearest respective background signal from the cell (b). In this example, a logarithmic scale is used to evidence the 2 orders of magnitude difference at maximum scattering of the particle (at $580 \mathrm{~nm}$ ). (B) An example of a single particle spectrum showing clearly periodic interferences (black line) when plotted against transition energy. A corresponding fitting function constituted of a sinusoid-modulated Gaussian function (as described in the main text) is displayed (red line).

field illumination (Figure 2B). After gold nanoparticle injection and mild agitation of the medium, the same cell is checked and illumination is changed to the supercontinuum white light. When a nanoparticle stops diffusing freely in the medium and comes into contact with the cell, tracking starts by scanning a zone of $2.5 \times 2.5 \times 3.5 \mu \mathrm{m}^{3}$ (300 lattice points) centered on the nanoparticle. This is achieved by using a piezo electric controlled stage (Nano LP-100, Mad City Laboratories.) synchronized with the ICCD. Scans were usually performed every $2 \mathrm{~min}$.

Spectrum Acquisition and Processing. Acquisition time was $0.1 \mathrm{~s}$ for each lattice point. Signals are subtracted from the average cell background before being divided by the incident light spectrum, in order to obtain scattering efficiency as a function of wavelength. The scattering peak is fitted as a Gaussian function modulated by a sinusoidal signal (cf. Figure 3 and following discussion section).

\section{Results and Discussion}

Spectral Characteristics. As the system is based on light scattering, it is important to consider first the background signal coming from fibroblast cells. An example of the signal can be seen in Figure 3A along with a signal from a nanoparticle. The main component of the scattering signal originates from different refractive index mismatches: (1) between membrane and extracellular medium (refractive indices being 1.37 for membrane and 1.33 for PBS), (2) between cytoplasm and organelles (typically 1.38 to 1.41 ), and (3) between cytoplasm and nucleus (same refractive index range as organelles). ${ }^{61,62}$ The second component is an interference modulation of the light scattering occurring under laser illumination. The possible explanation of the sinusoidal modulation is interferences of the multiple scattering lights from organelles and/or the membranes. These are consistent with the simulated angular variations of scattering by a model cell comprising cytoplasm, organelles, and nucleus reported by Drezek et al. ${ }^{62}$ Another important trend is that the absolute signal from a cell as well as the average period of interferences increases with the height from the depth bottom, which suggests that the interference between the scattering light from cell organelles and reflection light from the bottom of the dish attributes to the spectral modulation. As interference is of increasing modulation when going inside the cell, below the membrane, it helps to define the limit between cell and medium.

Occasional bright spots could be seen even when cells are examined in the absence of nanoparticles, which is naturally attributed to the organelles. According to Mourant et al. ${ }^{63}$ those constituting the main scatterers inside fibroblast cells are mitochondria ( $1-4 \mu \mathrm{m}$ length, $0.3-0.7 \mu \mathrm{m}$ diameter), lysosomes and peroxysomes $(0.3-0.7 \mu \mathrm{m}$ diameter), Golgi apparatus, and

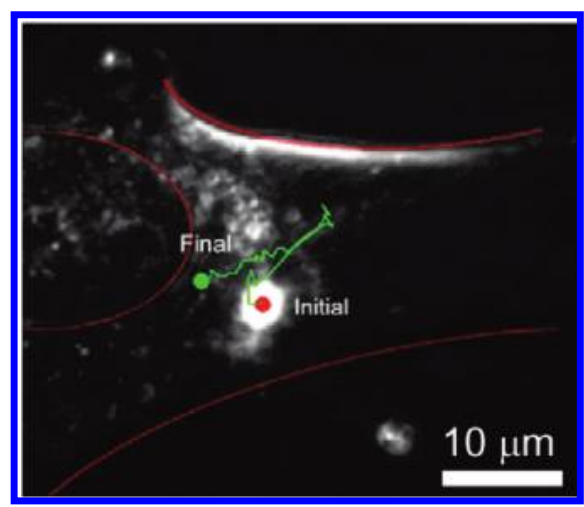

Figure 4. A dark field image of a fibroblast cell with a superimposed view of membrane and nucleus for maximum clarity. The path of a gold nanoparticle over an hour in the cell plane is also superimposed.

endoplasmic reticulum. Even though these scatterers have a wide size distribution, their average radius is estimated at $0.2 \mu \mathrm{m}$.

Panels A and B of Figure 3 show two typical signals from single gold nanoparticles in contact with cells. The background signal from cells, as illustrated in Figure 3A, is low, typically 2 orders of magnitude lower than the maximum scattering of the nanoparticle. Both spectra of the nanoparticles show the expected typical particle plasmon resonance band with its maximum in the 550-600 $\mathrm{nm}$ wavelength region, and these exhibit an interference modulation similar to the background signal from cells described above. These interferences were often observed for particles in contact or inside cells; when visible, the interference modulation and period were varied. The modulation ranged from 0 to 0.3 and the period ranged from $(10 \mathrm{~nm})^{-1}$ to $(40 \mathrm{~nm})^{-1}$. Although any definite correlation was not observed, as a trend, the modulation became smaller when the particle was located at higher position. To determine the plasmon resonance peak of the particles as follows, we took into account the interference as follows. When the scattering intensity was plotted against photon energy, $x=1 / \lambda$, where $\lambda$ is the wavelength of light, the spectral shape was best reproduced by the following equation:

$$
S_{\mathrm{E}}=A\left(\exp \left(\frac{\left(x-x_{0}\right)^{2}}{w}\right)+b \sin \left(\frac{2 \pi\left(x-x_{i}\right)}{T}\right)\right)
$$

Here a Gaussian function of amplitude $A$ and width $w$ is expressed as a function of $x(1 / \lambda)$, and the peak is situated at $x_{0}$. It is then modulated by a sinusoid of amplitude $b$ and period $T$, whose origin is $x_{i}$.

Spectral Tracking. To give a practical description of the experiments, a dark field image of a fibroblast cell with a superimposed typical nanoparticle trajectory can be seen in Figure 4. Further, relevant examples of tracking experimental results are displayed in Figures 5-7. Different behaviors were observed between these experiments and will be discussed in detail, after giving general comments on the tracking of experiment results.

From the time of contact between a particle and the cell membrane (drastic reduction in Brownian motion), the observed particles typically diffused about $5 \mu \mathrm{m}$ over an hour. No particular direction for the movement was observed, while in some cases the particles traveled toward or away from the cell nucleus. As a general trend, in most experiments, the depth of the particle decreased by about $1-2 \mu \mathrm{m}$, but in other cases an upward trajectory was observed. 

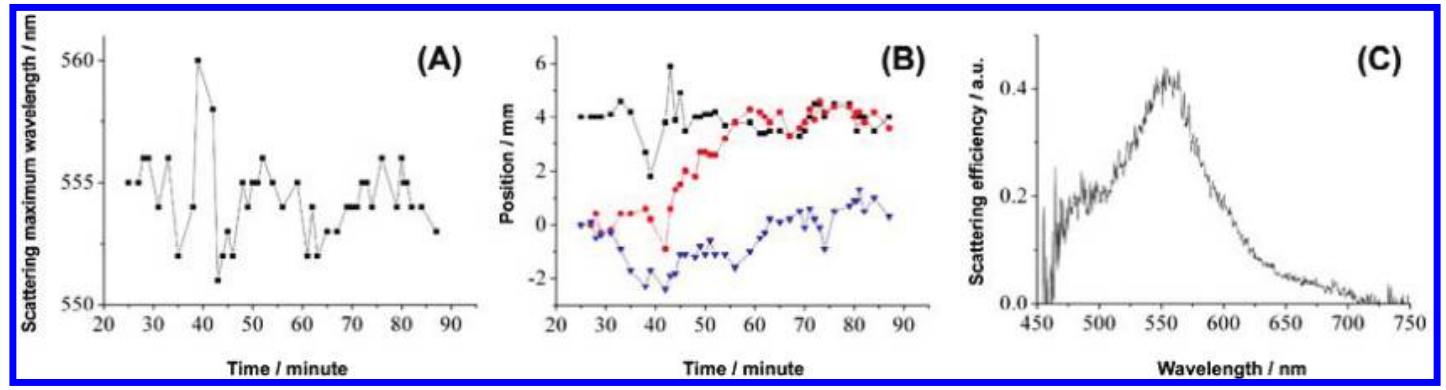

Figure 5. Tracking case I: (A) Scattering spectrum maximum wavelength evolution with time. (B) Particle position evolution with time ( $x$, $y$, and $z$ positions in red circles, blue triangles, and black squares, respectively); $x$ and $y$ positions are measured starting from the initial tracking position, while $z$ values are the distance from the upper surface of the dish. (C) Typical spectrum (at $52 \mathrm{~min}$ ).
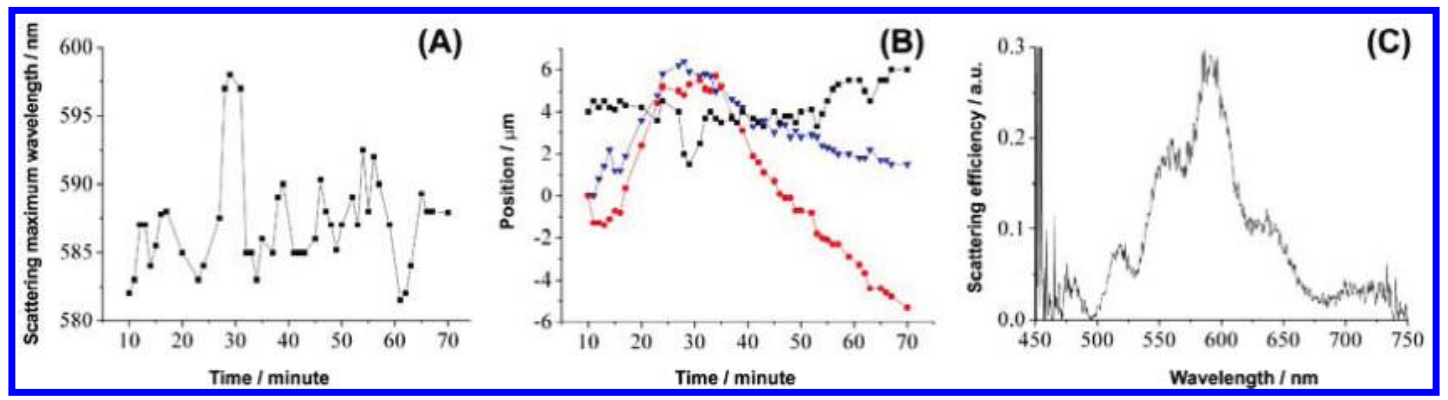

Figure 6. Tracking Case II: (A) Scattering spectrum maximum wavelength evolution with time. (B) Particle position evolution with time ( $x, y$, and $z$ positions in red circles, blue triangles, and black squares, respectively); $x$ and $y$ positions are measured starting from the initial tracking position, while $z$ values are the distance from the upper surface of the dish. (C) Typical spectrum (at $28 \mathrm{~min}$ ).

On the time scale of a minute or below, the changes of the maximum wavelength of the particle signal were typically of a few nanometers $(0-4 \mathrm{~nm})$. This could be attributed partially to variations of the laser focus point relative to the particle, since the light beam naturally displays some small spatial chromatic variations. Brownian motion of the particle in a short time range could be responsible for the spectral changes. The particle entering and then leaving the vicinity of cell organelles and membranes could be attributed to the rapid spectral changes, because the plasmon resonance peak wavelength of a gold nanoparticle is sensitive to refractive index change of the local environment. These variations constitute the effective experimental noise of our particle tracking trials. On the scale of 5 to 10 min, significant changes of the scattering wavelength maximum, intensity, as well as interference modulation, could sometimes be observed and related to biological phenomena as will be discussed below. Finally, on the scale of an hour, we sometimes observed a significant change the particle scattering properties.

Contrary to the reasonable wavelength accuracy in most of our experiments, the intensity of particle signals fluctuated by a factor of 2 . This could be explained by even slight defocusing from the very tight laser focal point compared to the particle position, and at the same time to local changes in the background scattering signal. Kyriacou et al ${ }^{49}$ performed real time imaging of silver nanoparticles of different sizes, in bacterial cells, under dark field halogen illumination. Fast imaging being recorded by a monochrome camera, color changes were not discussed, but authors reported a $10 \%$ decrease in intensity when particles entered the membrane, which was interpreted as the absorption of incident light from the bacteria and reduction in the scattering cross section due to the particle environment. In our case, the fibroblast cell has no absorption in the visible region and there was no such correlation; particles going deeper in the cell did not display a lower average intensity, nor were they of a dim appearance as was reported in their case.

As signal intensity is not a relevant parameter, we focused on the scattering maximum wavelength and its correlation to the position of the nanoparticle, as well as to the variations of interferences. One of the main behaviors observed is that some particles did not show any abrupt change in height, scattering maximum, and displayed weak interferences, from which we concluded that the particle never entered the cell in these experiments. The other behavior is that, when

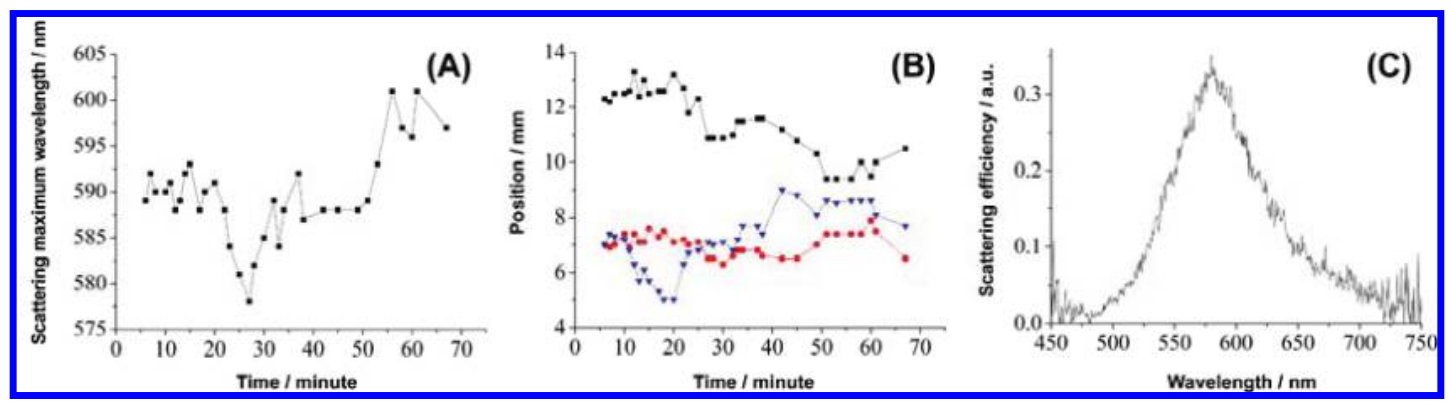

Figure 7. Tracking Case III: (A) Scattering spectrum maximum wavelength evolution with time. (B) Particle position evolution with time ( $x$, $y$, and $z$ positions in red circles, blue triangles, and black squares, respectively); $x$ and $y$ positions are measured starting from the initial tracking position, while $z$ values are the distance from the upper surface of the dish. (C) Typical spectrum (at $33 \mathrm{~min}$ ). 
SCHEME 1: Simplified Representation of Possible Endocytic Processes of a Gold Nanoparticle in a Fibroblast Cell ${ }^{a}$

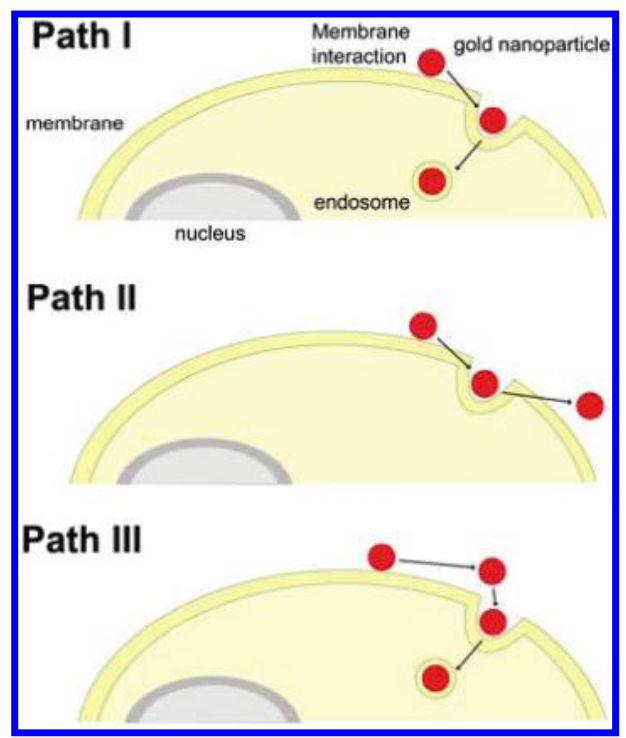

${ }^{a}$ After an initial contact, endocytosis of the particle (path I) notably through pinocytosis will enclose the particle in a lipid double layer, forming an early endosome. The particle can then be rejected out of the cell by the reverse process (path II), or the early endosome can grow by fusion of other vesicules to form a mature endosome, whose membrane is further from the particle (path III).

considerable shifts in the peak wavelength correlated with changes in the particle depth were observed, we suspected that a biological phenomenon occurred, which is presented in the following section.

Scattering Spectral Shifts Reflecting Changes in the Cell Local Environment. In tracking case I (Figure 5), the particle located at the cell periphery $(4 \mu \mathrm{m}$ height from the bottom of the dish) stayed at almost the same height during the whole observation time, and on average moved slowly along the cell, slightly away from the nucleus. During most of the experiment, interference modulation is very weak as can be seen on a typical spectrum in Figure 5C. According to the data, there was a singular event at $35 \mathrm{~min}$ : an abrupt decrease of height $(1.3 \mu \mathrm{m})$ that was correlated to a red shift (about $5 \mathrm{~nm}$ ) of the maximum wavelength in the scattering spectra of the nanoparticle. This was followed by some overshooting in position and then it returned to the initial state in a few minutes.

Case II (Figures 6) corresponds to the image displayed in Figure 4 . The particle height increasing during the last part of the tracking (coherent with the particle getting even closer to the top of the cell) was observed along with a small increase of the scattering peak wavelength over $1 \mathrm{~h}$. In this case interferences were common during the whole experiment, as can be seen in Figure 6. There was a singular event at $28 \mathrm{~min}$, whose characteristics are very similar to what was observed in case I: a shift of scattering maximum wavelength to the red $(12 \mathrm{~nm})$ was observed along with a decrease of the depth of the particle, followed by a return to its initial state after $5 \mathrm{~min}$.

In tracking case III (Figure 7), the particle was near the top of the cell (12.5 $\mu \mathrm{m}$ from the bottom of the dish) at a starting position. During the experiment the particle apparently stayed in the region above the nucleus, which is consistent with a weak interference modulation and a relatively small wavelength period of $15 \mathrm{~nm}$. We consider that the largest scattering maximum wavelength $(590 \mathrm{~nm})$ suggests the biggest particle in the three cases described. Two events should be noticed: (1) an event occurring in the middle of the experiment $(25 \mathrm{~min})$ shows the same diminution of particle position but this time a significant decrease in the scattering maximum wavelength was observed and (2) the final increase of the scattering maximum of the particle $(50 \mathrm{~min})$, along with an important diminution of the particle position resembling what was observed in the first two cases, but there is no return to its initial state.

Scheme 1 represents a possible explanation of some of these observations; after an initial contact there could be engulfment of the particle in the cell either through pinocytosis or by entering a small caveolae ${ }^{64}$ At this point of particle endocytosis, the particle would be tightly surrounded by a membrane layer (path I). This phase could be followed by rapid efflux of the particle out of the cell (path II) in the following minutes, because of its exogenous character. Short endocytocis/exocytosis cycles have indeed been reported by $\mathrm{Xu}$ et al. ${ }^{48}$ The $6-12 \mathrm{~nm}$ shift in the scattering maximum we observed in cases I and II could be explained by an increase in the local dielectric constant upon penetration of the particle in the membrane of the cell (or early endosome), because it may be closely packed by a lipid double layer. This consideration is supported by a simple numerical calculation of the scattering spectrum of lipid-coated nanoparticles. We calculated the scattering spectrum of a 80-nm gold sphere coated with a 5-nm lipid double layer (refractive index of 1.44) in PBS buffer (refractive index of 1.33). A red shift of $5 \mathrm{~nm}$ from the spectral peak of bare nanoparticle is expected in the theoretical prediction based on Mie theory. The apparent return to the initial state could be explained by the opposite phenomenon, i.e. the vesicle fusing back with the membrane, or even by an escape of the particle from a vesicle before full endocytosis. Another possibility, probably less likely, could nonetheless be considered (path III in Scheme 1): if other early endosomes fuse into the one containing the nanoparticles, the lipid double layer radius will increase, and its influence on the plasmon resonance of the nanoparticles disappears. It can be noted that the first hypothesis (endocytosis followed by exocytosis) is more consistent with the observed height changes, because it is identical after and before the spike. As for case III, the transitory blue shift observed should correspond to a decrease of the surrounding refractive index. The particle being initially on the membrane, a transitory repulsion of the particle from the cell could be considered, while the last red shift may correspond to an endocytic event as described for cases I and II.

To conclude on this series of experiments, in all cases, there was a definite trend between the particle scattering maximum position and the intensity of interferences. Events corresponding to a change in height of $1-2 \mu \mathrm{m}$ along with an increase of cell background signal and interferences could be interpreted as endocytic processes. The extent of the wavelength shifts was of the order of 5-10 nm, just above experimental noise in vivo. Variability of results can be explained by the uniqueness of each experiment; single cell studies have the drawback that every cell can respond in a different fashion according to its own history. All these phenomena would be cleared by the present systems and 3-dimensional tracking method.

\section{Conclusions}

In this study, we have demonstrated the in vivo spectral imaging and tracking possibilities of the confocal Rayleigh scattering spectroscopic imaging system developed in our group. To our knowledge, we were the first to monitor the change of spectral properties of individual gold nanoparticles due to interaction with living cells, which could be linked to the formation of lipid layers close to the particle surface during 
endocytotis and exocytosis processes. Also, as some phenomena observed in our experiments were on the minute time scale, improvements on the time resolution of the present spectroscopy, for example, on the millisecond scale, would give valuable information to help in the understanding of molecular aspects of the biological system. It can also be argued that particles other than spherical gold may show a more important shift. Although we believe that gold is indeed a good choice compared to silver because of its lower cytotoxicity, even though plasmon shifts are less wide, ${ }^{19}$ nonspherical shapes (such as nanoshells or nanotriangles, for example) would help to get a stronger response to environment changes. Also, it would be interesting to monitor the formation of particle dimers in vivo, since an intense scattering wavelength shift is expected but would necessitate a much higher concentration. Since metal nanoparticles are becoming more and more promising as therapeutics and detection agents, the present results of this work could be a physical chemistry base for more biology-oriented studies in other research groups.

Acknowledgment. G.L. thanks the Japanese Society for the Promotion of Science for granting the research fellowship and support leading to this work. The present work is partly supported by KAKENHI (the Grant-in-Aids for Scientific Research) on Priority Area "Strong Photon-Molecule Coupling Fields" (Area No. 470, No. 19049011) to T.A. and on Priority Area "Molecular Nano Dynamics (2004-2006)" from the Ministry of Education, Culture, Sports, Science and Technology of Japan (MEXT) and a KAKENHI (S) grant (No. 18106002) to H.M. from the Japan Society for the Promotion of Science (JSPS), the MOE-ATU Project (National Chiao Tung University) of the Ministry of Education, Taiwan to H.M., and the National Science Council of Taiwan (0970027441) to H.M.

Supporting Information Available: Numerical calculation for the scattering spectrum of a 80-nm gold sphere coated with 5-nm lipid double layer (refractive index of 1.44). This material is available free of charge via the Internet at http:// pubs.acs.org.

\section{References and Notes}

(1) Kreibig, U.; Vollmer, M. Optical Properties of Metal Clusters; Springer: Berlin, Germany, 1995.

(2) Yguerabide, J.; Yguerabide, E. E. Anal. Biochem. 1998, 262, 137.

(3) Yguerabide, J.; Yguerabide, E. E. Anal. Biochem. 1998, 262, 157.

(4) Kawata, S.; Masuhara, H. Plasmonics: From Fundamentals to Applications; Elsevier: Amsterdam, The Netherlands, 2006.

(5) Link, S.; El-Sayed, M. J. Phvs. Chem. B 1999, 103, 4212.

(6) Schultz, S.; Smith, D. R.; Mock, J. J.; Schultz, D. A. Proc. Natl. Acad. Sci. U.S.A. 2000, 97, 996.

(7) Sönnichsen, C.; Geier, S.; Hecker, N. E.; Von Plessen, G.; Feldman, J.; Ditlbacher, H.; Lamprecht, B.; Krenn, J. R.; Aussenegg, F. R.; Chan, V. Z.-H.; Spatz, J. P.; Möller, M. Appl. Phvs. Lett. 2000, 77, 2949.

(8) Mock, J. J.; Barbic, M.; Smith, D. R.; Schultz, D. A.; Schultz, S. J. Chem. Phvs. 2002, 116, 6755.

(9) Bohren, C. F.; Huffman, D. R. Absorption and Scattering of Light by Small Particles; John Wiley \& Sons: New York, 2002. L76.

(10) Itoh, T.; Asahi, T.; Masuhara, H. Jpn. J. Appl. Phvs. 2002, 41,

(11) Kelly, K. L.; Coronado, E.; Zhao, L. L.; Schatz, G. C. J. Phys. Chem. B 2003, 107, 668.

(12) Link, S.; El-Sayed, M. A. Annu. Rev. Phvs. Chem. 2003, 54, 331

(13) Daniel, M.-C.; Astruc, D. Chem. Rev. 2004, 104, 293.

(14) Jain, P. K.; Lee, K. S.; El-Sayed, I. H.; El-Sayed, M. A. J. Phvs. Chem. B 2006, 110, 7238 .

(15) Sendroiu, I. E.; Mertens, S. F. L.; Schiffrin, D. J. Phvs. Chem. Chem. Phvs. 2006, 8, 1430.

(16) Elghanian, E.; Storhoff, J. J.; Mucic, R. C.; Letsinger, R. L.; Mirkin, C. A. Science 1997, 277, 1078.
(17) Storhoff, J. J.; Elghanian, R.; Mirkin, C. A.; Letsinger, R. L. Langmuir 2002, 18, 6666.

(18) Riboh, J. C.; Haes, A. J.; McFarland, A. D.; Ranjit Yonzon, C.; Van Duyne, R. P. J. Phvs. Chem. B 2003, 107, 1772.

(19) Haes, A. J.; Hall, W. P.; Chang, L.; Klein, W. L.; Van Duyne, R. P. Nano Lett. 2004, 4, 1029.

(20) Haes, A. J.; Chang, L.; Klein, W. L.; Van Duyne, R. P. J. Am. Chem. Soc. 2005, 127, 2264.

(21) Rosi, N. L.; Mirkin, C. A. Chem. Rev. 2005, 105, 1547.

(22) Liao, H.; Hafner, J. H. Chem. Mater. 2005, 17, 4636.

(23) Katz, E.; Willner, I. Angew. Chem., Int. Ed. 2004, 43, 6042.

(24) Sokolov, K.; Follen, M.; Aaron, J.; Pavlova, I.; Malpica, A.; Lotan, R.; Richards-Kortum, R. Cancer Res. 2003, 63, 1999.

(25) El-Sayed, I. H.; Huang, X.; El-Sayed, M. A. Cancer Lett. 2006, $239,129$.

(26) El-Sayed, I. H.; Huang, X.; El-Sayed, M. A. Nano Lett. 2005, 5, 829.

(27) Gobin, A. M.; O’Neal, D. P.; Watkins, D. M.; Halas, N. J.; Drezek, R. A.; West, J. L. Laser Surg. Med. 2005, 37, 123.

(28) Wang, Y.; Xie, X.; Wang, X.; Ku, G.; Gill, K. L.; O’Neal, D. P.; Stoica, G.; Wang, L. V. Nano Lett. 2004, 4, 1689.

(29) Loo, C.; Lowery, A.; Halas, N.; West, J.; Drezek, R. Nano Lett. 2005, 5, 709.

(30) Chen, J.; Wiley, B.; Li, Z. Y.; Campbell, D.; Saeki, F.; Cang, H.; Au, L.; Lee, L.; Li, X.; Xia, Y. Adv. Mater. 2005, 17, 2255.

(31) Sandhu, K. K.; McIntosh, C. M.; Simard, J. M.; Smith, S. W.; Rotello, V. M. Bioconj. Chem. 2002, 13, 3.

(32) Tkachenko, A. G.; Xie, H.; Coleman, D.; Glomm, W.; Ryan, J.; Anderson, M. F.; Franzen, S.; Feldheim, D. L. J. Am. Chem. Soc. 2003, 125,4700 .

(33) Tkachenko, A. G.; Xie, H.; Liu, Y.; Coleman, D.; Ryan, J.; Glomm, W. R.; Shipton, M. K.; Franzen, S.; Feldheim, D. L. Bioconi. Chem. 2004, 15,482 .

(34) Kawano, T.; Yamagata, M.; Takahashi, H.; Niidome, Y.; Yamada, S.; Katayama, Y.; Niidome, T. J. Controlled Release 2006, 111, 382.

(35) Takahashi, H.; Niidome, Y.; Yamada, S. Chem. Commun. 2005, 2247

(36) Chen, C. C.; Lin, Y. P.; Wang, C. W.; Tzeng, H. C.; Wu, C. H.; Chen, Y. C.; Chen, C. P.; Chen, L. C.; Wu, Y. C. J. Am. Chem. Soc. 2006, $128,3709$.

(37) Rosi, N. L.; Giljohann, D. A.; Thaxton, C. S.; Lytton-Jean, A. K. R.; Han, M. S.; Mirkin, C. A. Science 2006, 312, 1027.

(38) Niemeyer, C. M.; Simon, I. Eur. J. Inorg. Chem. 2005, 2005, 3641.

(39) Beissenhirtz, M. K.; Elnathan, R.; Weizmann, Y.; Willner, I. Small 2007, 3, 375 .

(40) Thanh, N. T. K.; Rosenzweig, Z. Anal. Chem. 2002, 74, 1624.

(41) Reinhard, B. M.; Siu, M.; Agarwal, H.; Alivisatos, A. P.; Liphardt, J. Nano Lett. 2005, 5, 2246.

(42) Raschke, G.; Kowarik, S.; Franzl, T.; Sonnichsen, C.; Klar, T. A.; Feldmann, J.; Nichtl, A.; Kurzinger, K. Nano Lett. 2003, 3, 935.

(43) Schmidt, T.; Schutz, G. J.; Baumgartner, W.; Gruber, H. J.; Schindler, H. Proc. Natl. Acad. Sci. U.S.A. 1996, 93, 2926.

(44) Daumas, F.; Destainville, N.; Millot, C.; Lopez, A.; Dean, D.; Salome, L. Biophvs. J. 2003, 84, 356.

(45) Saxton, M. J.; Jacobson, K. Annu. Rev. Biophvs. Biomol. Struct. 1997, 26, 373

(46) Levi, V.; Ruan, Q.; Gratton, E. Biophvs. J. 2005, 88, 2919.

(47) Holtzer, L.; Meckel, T.; Schmidt, T. Appl. Phvs. Lett. 2007, 90, 053902.

(48) Xu, X.-H. N.; Chen, J.; Jeffers, R. B.; Kyriacou, S. Nano Lett. 2002, 2,175 .

(49) Kyriacou, S. V.; Brownlow, W. J.; Xu, X. H. N. Biochemistry 2004, $43,140$.

(50) Murase, K.; Fujiwara, T.; Umemura, Y.; Suzuki, K.; Iino, R.; Yamashita, H.; Saito, M.; Murakoshi, H.; Ritchie, K.; Kusumi, A. Biophvs. J. 2004, 86, 4075 .

(51) Yelin, D.; Oron, D.; Thiberge, S.; Moses, M.; Silberberg, Y. Opt. Express 2003, 11, 1385

(52) Boyer, D.; Tamarat, P.; Maali, A.; Lounis, B.; Orrit, M. Science 2002, 297, 1160.

(53) Cognet, L.; Tardin, C.; Boyer, D.; Choquet, D.; Tamarat, P.; Lounis, B. Proc. Natl. Acad. Sci. U.S.A. 2003, 100, 11350.

(54) Dijk, M. A. v.; Tchebotareva, A. L.; Orrit, M.; Lippitz, M.; Berciaud, S.; Lasne, D.; Cognet, L.; Lounis, B. Phvs. Chem. Chem. Phvs. 2006, 8, 3486.

(55) Lasne, D.; Blab, G. A.; Berciaud, S.; Heine, M.; Groc, L.; Choquet, D.; Cognet, L.; Lounis, B. Biophys. J. 2006, 91, 4598.

(56) Lindfors, K.; Kalkbrenner, T.; Stoller, P.; Sandoghdar, V. Phvs. Rev. Lett. 2004, 93, 037401.

(57) Jacobsen, V.; Stoller, P.; Brunner, C.; Vogel, V.; Sandoghdar, V. Opt. Express 2006, 14, 405

(58) (a) Asahi, T.; Uwada, T.; Louit, G.; Masuhara, H. LEOS Summer Topical Meeting: IEEE Lasers and Electro-Optics Society: New York, 2008; 
art. no. 4590492, p 67. (b) Uwada, T.; Yamaguchi, A.; Asahi, T.; Masuhara, H. To be submitted.

(59) Chithrani, B. D.; Ghazani, A. A.; Chan, W. C. W. Nano Lett. 2006, 6,662 .

(60) Ellen E. Connor, J. M. A. G.; Catherine, J. M.; Michael, D. W. Small 2005, 1, 325 .

(61) Drezek, R. A.; Dunn, A.; Richards-Kortum, R. Appl. Opt. 1999, 38,3651 .
(62) Drezek, R. A.; Dunn, A.; Richards-Kortum, R. Opt. Express 2000, $6,147$.

(63) Mourant, J. R.; Freyer, J. P.; Hielscher, A. H.; Eick, A. A.; Shen, D.; Johnson, T. M. Appl. Opt. 1998, 37, 3586.

(64) Conrad, P. A.; Smart, E. J.; Ying, Y. S.; Anderson, R. G.; Bloom, G. S. J. Cell Biol. 1995, 131, 1421.

JP9018124 